\title{
Le journalisme au miroir de la littérature (à moins que ça ne soit l'inverse)
}

\author{
Bertrand Labasse
}

Université d'Ottawa

Ébranlant le vieux canon belle-lettriste - qui, à dire vrai, en a vu d'autres - , une décennie a suffi pour ériger la production journalistique en un « objet littéraire à part entière - au même titre que le drame romantique ou que tel roman de Balzac» (Vaillant, 2001, p. 7). Si besoin était, l'imposant foisonnement des colloques, ouvrages et articles qui s'en sont saisis depuis le début du siècle confirmerait amplement, par le nombre et par la valeur, combien cette transgression était nécessaire, tant pour l'histoire culturelle en général que pour la compréhension des œuvres littéraires elles-mêmes - qui, depuis le XIX ${ }^{\text {e }}$ siècle, se sont construites avec ou contre le journal, mais toujours par 
rapport à lui - et encore pour l'histoire de la presse, à qui les spécialistes de la littérature (ainsi qu'ils se présentaient sans détour) ont permis de redécouvrir les productions journalistiques non seulement comme des discours, des témoignages ou des indices ${ }^{1}$ mais aussi en tant que textes.

Pour autant, ces renouvellements n'ont pas aplani - ce serait mauvais signe - tous les problèmes qu'ils ont mis ou remis au jour. Un bilan récent (Vaillant, 2011) et un colloque plus récent encore (Héron et Thérenty, 2014) incitent à s'arrêter sur l'un d'eux.

Étant admise la flatteuse proposition initiale selon laquelle toute production journalistique peut, a priori, s'approcher comme une œuvre littéraire, l'équité la plus élémentaire suggère d'accorder à la littérature une légitimité réciproque dans l'ordre journalistique: tout texte d'écrivain paru dans un journal peut, a priori, s'approcher comme du journalisme. De là, un pas suffit pour accoler, dans la meilleure tradition de la littérature enfantine ${ }^{2}$, le titre de journaliste à des patronymes parfois évidents ("Colette journaliste», «Zola journaliste», etc.), parfois beaucoup moins. Or, en confrontant cette proposition à ses limites, le colloque «Cocteau journaliste» et l'ouvrage qui en découle ${ }^{3}$ pourraient en constituer le test.

C'est pourquoi, abandonnant toute idée de recension (« quinze contributions », «varié», « complet», «passionnera

\footnotetext{
1 « [...] en dépit de sa richesse et de sa diversité, l'histoire de la presse a jusqu'ici été surtout pensée en termes d'institution: la presse comme institution (histoire des titres et des entreprises), ses liens avec l'institution politique ou avec celles de la "sphère publique". " (Kalifa et Vaillant, 2004) 2 « Les pieds nickelés journalistes » (1961), « Mickey reporter» (1978), etc.

${ }^{3}$ Auquel on se référera dans la suite de cet article par les initiales CJ.
} 
les amateurs de Cocteau », etc.), cet article se propose de saisir cette occasion pour revenir sur la proposition d'Alain Vaillant : « À l'intérieur du couple presse / littérature, c'est évidemment la littérature qui pose les problèmes définitionnels les plus graves.» (p. 23) Au cours de cette étude, «théorique» en ce qu'elle appuie sur nombre de textes mais non sur un corpus défini ni même sur une période circonscrite, on adoptera un cheminement concentrique, partant des écrits de presse de Cocteau pour élargir l'examen à certains des "problèmes définitionnels » dont ils sont les révélateurs, l'étendre encore à la textualisation du réel en général, puis le dilater jusqu'à englober des questions aussi familières (et décourageantes) que celle de la littérarité et de l'épistémologie des études littéraires, dont on soutiendra que l'une et l'autre pourraient retirer quelques éclairages utiles de la prise en compte des textes journalistiques « ordinaires ».

Il va sans dire qu'un tel parcours impose de sérieux raccourcis et interdit du même coup toute prétention à construire un dispositif démonstratif implacable: il s'inscrit donc plutôt dans la lignée générique des «réflexions» et "propositions », empruntant là une forme un peu désuète d'un point de vue scientifique mais non dépourvue de vertus heuristiques.

\section{Une production « journalistique »?}

Abstraction faite de la détestation que Cocteau manifestait inlassablement à l'égard de la presse ${ }^{4}$, la multiplicité de ses

4 «Quoi ? Le poète aurait lutté des années contre le journalisme, contre ses séides [...] pour se voir in fine qualifié à son tour d'un terme infamant? 
contributions à celle-ci n'a rien pour surprendre d'un polygraphe avide d'explorer à peu près tous les champs et supports de l'expression créative, jusqu'à la radio et la publicité. La majorité de ses écrits de presse s'inscrit toutefois dans la tradition des textes d'esprit ou de jugement culturel, la sphère équivoque de la chronique, qui connaîtra son apogée avec les feuilletons de La Presse de Girardin mais qui accompagnait l'écriture d'information depuis sa naissance ou presque sans pour autant se confondre avec elle ${ }^{5}$. La tradition contre laquelle s'est produite la «révolution à laquelle nous assistons depuis vingt ans dans le journalisme et qui se résume ainsi : décadence de la chronique et progrès du reporter » (Filon, 1889, p. 378). La tradition, en somme, vis-à-vis de laquelle s'est élaboré ce que, depuis la fin du XIXe siècle, on tient pour du journalisme :

Le Matin ne publiera pas de feuilleton. C'est là un mode de journalisme qui ne saurait trouver place dans un journal débordant d'informations et de nouvelles. (Le Matin, 1884, p. 1)

Indépendamment de leur valeur propre, ce ne sont donc pas ces textes qui interrogent le mieux ce qu'il peut bien y avoir de journalistique dans un journal : s'il suffisait d'y prendre place pour s'y assimiler, les mots croisés, les bandes dessinées et même la publicité ne sauraient être distingués des articles d'actualité (mais songe-t-on à publier «General motors journaliste » ou « Coca cola journaliste »?).

Il ne s'agit évidemment pas de tracer autour du journalisme une frontière qui prétendrait en expulser la

Journaliste ne surgit jamais en effet sous sa plume que par marque de dépréciation. », résume Guy Ducrey (CJ, p. 157) en introduction à un florilège de citations dans ce sens.

5 Les différences de contenu, de style et même de posture entre la Gazette et le

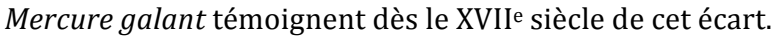


chronique ou la critique, genres traditionnellement métis, mais plutôt de se concentrer ici sur une série d'articles plus inattendue et, surtout, plus intrigante, car plus typiquement journalistique dans son objet: le tour du monde de Cocteau pour le compte de Paris-Soir.

Archétype de la presse à grand tirage, le quotidien, dont Camus, qui y fut brièvement secrétaire de rédaction, dénonça «l'abject esprit de midinette [...]. La sentimentalité, le pittoresque, la complaisance, tous ces refuges visqueux où l'homme se défend dans une ville si dure à l'homme » (1962, p. 212), était aussi éloigné d'une revue culturelle qu'il est possible de l'être, mais il appréciait fort les contributeurs célèbres (Mac Orlan, Saint-Exupéry, etc.) et les récits exotiques. La proposition de Cocteau d'effectuer avec son ami Marcel Khill, dont c'était l'idée, un voyage sur les traces et dans les délais des personnages du «Tour du monde en 80 jours » de Jules Verne ne pouvait donc manquer de le séduire.

Des navires en partance, des palaces coloniaux, des péripéties assurées et un grand journal pour les raconter : toute l'imagerie populaire du journalisme au long cours se trouve ainsi convoquée. Mais, pour paré qu'il soit de certains des attributs les plus colorés du grand reportage, ce récit doit-il vraiment être considéré comme tel? Qu'une chercheuse aussi incontestable que Marie-Éve Thérenty, spécialiste s'il en est de l'étude littéraire du journalisme, y voie «une forme de reportage exemplaire, canonique » (CJ, p. 211), donne un poids certain à cette hypothèse. De fait, ainsi qu'elle le relève, le tour du monde de Cocteau s'inscrit «dans un dialogue intertextuel avec le genre [du grand reportage] tel qu'il se construit à l'époque » (CJ, p. 206). Cocteau lui-même, dans son premier 
article $^{6}$, évoque le projet de «poursuivre notre timide essai de reportage $^{7}[\ldots]$ et de prendre le large, n'importe lequel » (TdM, p. 18).

Pourtant, dès ce premier article, le doute s'insinue. Si le reportage y est allusivement mentionné - surtout comme une occasion «de prendre le large»-, c'est à de toutes autres influences que se réfère le plus explicitement Cocteau :

Dès le départ, nous devions prendre le rythme des familles Perrichon et Fenouillard [...]. Ces personnages de Töpffer et de Christophe, plus encore que ceux de Verne, furent à l'origine de cette poésie amoureuse qui nous habite depuis l'enfance et nous met le diable au corps ${ }^{8}$. Le vrai Japon, c'est madame Fenouillard et ses filles, grisant les gardes pour délivrer leur époux et père en perçant la cloison de papier [...]. Car les enfants rêvent sur ces épopées burlesques. Ils les déforment à leur usage et ils y puisent les éléments de féeries profondes. (p. 19)

Joignant - si l'on peut dire - le geste à la parole, l'auteur commence le récit de ses tribulations par une ridicule confusion d'horaires de train et une course éperdue dans un entrechoquement de sacs :

Si je vous raconte ces détails, c'est pour vous faire comprendre cette impossibilité française de se mettre en branle sans alpenstocks, sans marche pied pris d'assaut, sans billets cherchés dans toutes les poches et sans paquets qui tombent. (p. 20)

\footnotetext{
6 Toutes les citations du «Tour du monde en 80 jours » de Cocteau (désigné par « TdM » dans la suite de cet article) sont issues de l'édition de 2006.

7 Le premier essai, «timide » en effet, était le récit d'une promenade en bateau avec Marcel Khill, publié - sous le titre «Retrouvons notre enfance » — par Paris-Soir en août 1935.

8 Il est difficile de ne pas remarquer cette allusion à Raymond Radiguet, mais on peut hésiter à y voir plus qu'une évocation de circonstance.
} 
Un alpenstock pour sauter de trains en bateaux? Alors que les reporters professionnels se dispenseraient volontiers à bord d'un aussi encombrant bâton de montagne (Gaston Leroux disait justement d'un de ses confrères qu'il «fait le tour du monde sans un bâton» [1901, p.1]), c'est en revanche l'accessoire traditionnel des voyageurs rocambolesques, comme Tartarin de Tarascon et, plus tard, M. Dumollet.

Entrepris dans de telles dispositions, le voyage de Cocteau semble donc moins s'inspirer de la tradition du reportage qu'annoncer les débuts d'un nouveau type de contenu médiatique: opportunément publié en août 1936, l'année même des premiers congés payés, il préfigure d'une certaine façon ces longs délassements d'été, fictionnels ou non, avec lesquels les journaux agrémenteront les vacances de leurs lecteurs au plus creux de l'actualité.

Mais Cocteau étant Cocteau, qui prétendrait assigner ou refuser un statut à son récit s'exposerait à se voir aussitôt contredit par celui-ci. Dès son second texte, en effet, le poète, loin de tout badinage, administre une véritable leçon d'écriture journalistique, portant celle-ci à un niveau que peu de professionnels, avant ou après lui, parviendront à approcher. La leçon n'est certes pas dans la méthode : traverser hâtivement une ville, en taxi et de nuit, avec pour seule source d'information le murmure des fontaines, n'est pas connu comme la façon la plus rigoureuse d'inventorier la réalité. La leçon est dans le regard et dans la main, dans la capacité à saisir au clair de lune une Rome silencieuse, pétrifiée par la dictature mussolinienne comme par le poids de ses propres monuments, et, en une poignée de lignes seulement, à la faire ressentir avec une intensité saisissante. 
Une source d'inspiration pour le journalisme ? Sans aucun doute. Un modèle ? On frémit d'y penser. Sous un titre éloquent — «Croisière vers le peu de réalité »-, l'examen par Michel Coulomb de la séquence asiatique du récit de Cocteau y relève avec une indulgente cruauté tout ce que ces comptes rendus doivent à la «capacité fabulatrice » (CJ, p. 152) du poète. Audelà des détails suspects et des interprétations hasardeuses, une invention frappe par son ampleur, celle d'une métropole entière, pas moins, qui évoque vaguement Kuala Lumpur sans lui correspondre: «non, décidément, Cocteau n'y a jamais mis les pieds » (p. 154).

Les nombreux écarts avec la vérité qui parsèment les articles du Tour du monde - y compris le récit de son exaltante rencontre avec Charlie Chaplin ${ }^{9}$ - entretiennent la perplexité : est-il donc plus «journaliste» qu'il n'est sinologue ou égyptologue, lui dont les exégèses des coutumes chinoises ou des rites pharaoniques effareraient sans doute les savants les plus conciliants? S'il est heureusement inutile de se prononcer en la matière, c'est que Cocteau lui-même a apporté la réponse dans son discours de réception à l'Académie :

Messieurs, lorsque j'admire un peintre, on me dit: «Soit, mais ce n'est pas de la peinture. » Lorsque j'admire un musicien, on me dit: «soit, mais ce n'est pas de la musique.» Lorsque j'admire un dramaturge, on me dit: « soit, mais ce n'est pas du théâtre. » Lorsque j'admire un sportif, on me dit : «soit, mais ce

\footnotetext{
${ }^{9}$ Admettons sans trop de réticences qu'il a bien existé un acteur et réalisateur nommé Charlie Chaplin. Et que Cocteau l'a bien rencontré à bord du Karoa, l'Américain témoignant lui-même du fait dans ses mémoires. Mais la coïncidence entre les deux récits s'arrête quasiment là : « Le matin suivant, je me promenais seul sur le pont. Tout à coup, à mon horreur, Cocteau apparut, tournant un angle à bonne distance. Mon Dieu! Je cherchais hâtivement une voie d'évasion lorsqu'il m'aperçut et, à mon soulagement, s'élança à travers la porte du grand Salon. » (Chaplin, 1964/2012, p. 381, notre traduction)
} 
n'est pas du sport. » (C'est ce que j'entendais après chaque match d'Al Brown.) Et ainsi de suite. Mais alors, demandais-je : «Qu'est-ce que c'est? » Mon interlocuteur hésite, l'œil dans le vague et murmure : « je ne sais pas... C'est autre chose. »

J'ai fini par comprendre que cet autre chose était, somme toute, la meilleure définition de la poésie. (1960, p. 148)

Ainsi son Tour du monde et ses divers écrits pour la presse ne permettent-ils de réserve - «soit, mais ça n'est pas du journalisme » - qu'au prix d'une invocation, piteuse en effet, de cet « autre chose » que le poète revendique.

\section{Le reportage en son miroir}

Le legs le plus fascinant de Cocteau au journalisme ne réside évidemment pas dans sa méthode, mais il ne se trouve peutêtre même pas dans son écriture. Il tient à ce que le poète, qui s'y connaît en miroirs, présente au journalisme en général, et au grand reportage en particulier, des reflets énigmatiques d'euxmêmes où se brouille ce qu'ils sont, ne sont pas ou pourraient être. En d'autres termes, ceux d'Alain Vaillant, il leur pose avec nonchalance « les problèmes définitionnels les plus graves ».

Parmi les comptes rendus lointains dont Paris-Soir et ses concurrents de l'entre-deux-guerres étaient si friands, Pierre Albert prend soin de distinguer «les grands reportages à l'occasion des multiples crises et guerres qui agitaient le monde, les enquêtes [... et ...] les récits de voyage teintés d'exotisme » (1972, p. 478). Cette dernière catégorie ne paraît pas seulement convenir plus aisément que la première aux souvenirs rapportés par Cocteau; elle s'inscrit aussi dans une tradition de presse plus ancienne. C'est en effet dès la première moitié du XIXe siècle, rappelle Sylvain Venayre, que 
[...] se diffusa progressivement la pratique consistant, pour le journal, à financer le voyage d'un écrivain sur un lieu dont il devait rapporter un récit, lequel, écrit à la première personne et soumis à l'exigence du code romantique de "l'impression", n'en prétendait pas moins dire la vérité sur ce qu'il avait vu. (2007, p. 50)

Le distinguo paraît évident : la typicité du reportage — grand ou non - l'associe depuis ses origines à une quête extrinsèque, celle de la nouvelle, qui le suscite (comme les guerres de Crimée et de sécession, premiers terrains des envoyés spéciaux) ou qu'il construit lui-même en se rapprochant de l'«enquête» pour dévoiler une réalité méconnue (le bagne, les marchés d'esclaves, la Russie des soviets, etc.). Par contraste, un périple n'ayant d'autre objet que la contemplation d'un pittoresque dépourvu d'«actualité », voire sans autre sujet que lui-même, s'apparenterait plus à ces autoportraits de touristes en vacances dont les pyramides ou le Taj Mahal ne forment que l'arrière-plan.

Or, rien ne peut mieux ébranler cette dichotomie qu'un poète en partance pour le tour du monde. En effet, le défi de Jules Verne (dont l'origine fictionnelle réside, du reste, dans un article de press $\mathrm{e}^{10}$ ) avait, bien avant Cocteau, inspiré nombre de journaux et de journalistes. Précédant le poète, des reporters aussi indiscutables que Nellie Bly, du New York World, ou Gaston Stiegler, du Matin (pour ne citer qu'eux), avaient entrepris le voyage sans manifester le moindre doute sur le caractère « journalistique » de leur initiative :

L'idée m'en est venue un dimanche. J'avais passé la majeure

\footnotetext{
10 Rappelons que, dans le roman, un article du Morning Chronicle affirmant que 80 jours suffisent désormais pour faire le tour du monde occasionne la controverse, puis le pari, qui lanceront les héros autour de la terre, ce qui ramène leurs aventures à une sorte de facts checking poussée à l'extrême.
} 
partie de la journée et la moitié de la nuit à essayer en vain de trouver quelque idée pour un article de journal. C'était mon habitude d'imaginer des sujets le dimanche et de les soumettre à l'approbation ou la désapprobation de mon rédacteur en chef le lundi. (Bly, 1890/2009, p. 5, notre trad.).

Il faut dire actuellement, en 1901, au début de ce siècle, en combien de jours se fait le tour du monde. Est-ce en quarante, en quarante-cinq, en cinquante jours? Nous ne le savons pas d'une façon précise. Il n'y a qu'une manière sérieuse d'acquérir cette notion, c'est d'aller la chercher, c'est de faire le trajet soimême. La direction du Matin a compris l'intérêt de la question et m'a chargé de la résoudre sur place. (Stiegler, 1901a, p. 1).

Pourtant, Nellie Bly sera, plus encore que Cocteau, son propre sujet, et elle se souciera encore moins que lui de décrire le monde qu'elle traverse. Quant à Gaston Stiegler, ses impressions (contraintes, il est vrai, par la brièveté des étapes et le coût du télégraphe) sont parfois si sommaires qu'elles paraîtraient plates sur la carte postale de n'importe quel vacancier: "Le temps est très beau. Moscou, sous sa robe multicolore, est tout éclatant au soleil. Je me promets une pérégrination charmante. » (Stiegler, 1901b, p. 1) Les lecteurs de son livre pourront, plus tard, savourer la réelle qualité de son regard ${ }^{11}$, mais ce sont bien ses dépêches succinctes et, surtout, sa victoire sur ses concurrents qui lui vaudront, sous la

\footnotetext{
11 Passant du journal au livre, le journaliste ne manquera pas, par exemple, de développer tout ce qu'il avait condensé dans l'adjectif «multicolore »: «À Moscou tout est couleur et mouvement. Cette ville, où il n'y a pas de cafés et où la vie publique se réduit à peu de chose, est pourtant une des plus animées du monde, et serait une des plus gaies si le peuple y avait de la bonne humeur et de l'entrain. Excepté les tons gris et neutres de chez nous, on y voit toutes les teintes, les plus variées et les plus claires, des couvents rouges, des casernes jaunes, des églises d'azur, des maisons roses, et sous le soleil de l'été elle produit l'effet d'une ville du midi. Mais les gens, lourds et méditatifs, la tête penchée, ont l'air de promener je ne sais quel deuil dans une salle de fête. » (Stiegler, 1901c, p. 37)
} 
plume emphatique de Gaston Leroux, les titres de "premier reporter du monde» (Leroux, 1901b, p.1) et de "premier journaliste de la terre » (ibid., p. 2).

Aussitôt le départ donné, ces deux randonnées s'étaient éloignées à grandes foulées de l'idéal journalistique. C'est que, dès leur annonce, l'un et l'autre de ces projets avaient suscité des initiatives rivales. Contre le New York World et Nellie Bly, le magazine Cosmopolitan décide d'aligner une autre journaliste, Elizabeth Bisland. Contre Le Matin et Gaston Stiegler, le Journal lance le non moins chevronné Henri Turot, rendant caduque la noble déclaration du premier : " J'avertis tout de suite le lecteur que je ne voyage pas pour gagner un pari. » (Stiegler, 1901a, p. 1) Du moins demeure-t-on dans les deux cas entre professionnel(le)s de bonne compagnie. Mais le second duel, celui de 1901, tourne à la farce lorsque William Randolph Hearst s'y invite à son tour. Le magnat de la presse populaire américaine ne dépêche pas un reporter aguerri mais trois concurrents adolescents, dont chacun représente l'un de ses journaux (Chicago, New York, San Francisco) après avoir été élu par les professeurs des écoles locales. Un concours de pronostics destiné aux lecteurs achève d'enlever tout vernis de respectabilité journalistique à cette course publicitaire que Le Matin prend pourtant assez au sérieux pour s'indigner des tricheries des Américains.

N'eut été Cocteau, un voile d'insouciance aurait facilement couvert ce moment d'égarement du reportage au long cours. Mais le voyage de Cocteau est là et interroge : en quoi tiendrais-je moins du grand reportage que les récits du «premier reporter du monde» ou de cette Nellie Bly que célèbrent tant les écoles de journalisme? 
La question est d'autant plus sérieuse que, pour Marc Martin, c'est justement la répétition, désormais plus facile, des périples des reporters par des écrivains qui annonce la fin de l'âge d'or du grand reportage :

Faire le voyage déjà fait par un reporter, n'est-ce pas le signe que l'on n'est plus au moment où il faut percer les mystères des pays traversés ? [...] Le changement apparaît de manière encore plus significative quand c'est Jean Cocteau qui effectue pour Paris-Soir, du 29 mars au 19 juin 1936, ce qu'il dénomme "Mon premier Tour du Monde en 80 jours » [...]. En se plaçant du côté du journalisme de presse, on entrevoit déjà la mise en cause du métier même de grand reporter dont les effets éclateront au cours des années 1950. (2007, p. 127-128)

\section{Fictions du « grand » reportage}

L'embarras que suscite la notion de grand reportage n'est certes pas né de Cocteau et du livre qu'il vient de susciter, mais ceux-ci le ravivent opportunément. Les journalistes eux-mêmes en sont généralement venus à éviter cette locution, qu'ils laissent aux organisateurs de festivals, aux éditeurs de recueils... et au grand public. Certes, le titre de "grand reporter » correspond encore, en France, à un échelon salarial des conventions collectives, titre flatteur mais incertain que l'on attribue à ceux qui, leurs aptitudes établies, refusent d'abandonner le terrain pour les responsabilités éditoriales. Mais cette désignation ne circonscrit pas plus le «grand reportage» que celle de danseur étoile n'impose l'existence d'une « danse étoile » : «Disons que la qualification vaut surtout pour un organigramme [...]. Aujourd'hui, il n'y a pas plus de petits ou de grands reporters qu'il n'y a de petit ou de grand reportage.», tranche le manuel d'une école professionnelle 
réputée (de Broucker, 1995, p. 160). Tous conviennent, en tout cas, que ce n'est pas une question de distance, ni de risques encourus. C'est... C'est... "C'est autre chose!», soufflerait Cocteau.

Réputé trivial ou insoluble dans son champ d'origine, ce problème définitionnel n'en a pas moins été réinvesti par les spécialistes de la littérature, pour qui les questions de genre ne sauraient être prises à la légère. La démarche, comme il se doit, part des textes eux-mêmes et excelle à relever, tant «avant 1914 » (Thérenty 2006) qu'«au cœur des années trente» (Boucharenc, 2004), des caractères communs à beaucoup d'entre eux, comme la mise en scène du reporter et des aléas qu'il surmonte, l'authentification du récit par le témoignage de la chose personnellement vue et entendue, ou encore la relation presque épistolaire que le reporter établit avec le lecteur à qui il s'adresse.

Il va toutefois sans dire que les inductions textuelles, si elles dessinent bien une certaine typicité générique, ne peuvent fournir de caractère suffisant ou même nécessaire: elles ne permettraient pas plus d'exclure l'Itinéraire de Paris à Jérusalem de Chateaubriand ${ }^{12}$ que d'inclure les nombreux reportages ne recourant pas à la première personne, voire ceux d'Albert

\footnotetext{
12 « Je suivois, à peu près armé comme le janissaire, portant de plus un fusil de chasse $[\ldots . \mathrm{N}]$ ous grimpions au grand trot les montagnes, et nous les descendions au galop, à travers les précipices : il faut prendre son parti : les Turcs militaires ne connoissent pas d'autre manière d'aller, et le moindre signe de frayeur, ou même de prudence, vous exposeroit à leur mépris. Vous êtes assis, d'ailleurs, sur des selles de Mameloucks, dont les étriers larges et courts vous plient les jambes [...]. Le soir on arrive quelquefois à un kan, masure abandonnée où l'on dort parmi toutes sortes d'insectes et de reptiles sur un plancher vermoulu. On ne vous doit rien dans ce kan [...] : c'est à vous de vous procurer des vivres comme vous pouvez. » (Chateaubriand, 1812, p. 87-89)
} 
Londres sur le bombardement de la cathédrale de Reims (que pourrait disqualifier l'emploi du « nous » de distanciation).

Force a donc été de recourir à des considérations extratextuelles. La plus évidente est le fait d'être commandité par un journal, " capitale si l'on veut pouvoir distinguer toute une littérature de voyage [...] d'un reportage au sens journalistique du terme» (Boucharenc, 2004, p. 74). Mais, comme le souligne aussitôt cette auteure, «la commande d'un quotidien d'information ne suffit pas à donner toutes les garanties de l'authentique reportage » (p. 76), et d'en donner comme exemple, justement, précisément... le tour du monde de Cocteau! De même, la référence à une écriture sur le vif, inscrite dans la temporalité de ce qu'elle rapporte, peut trouver de nombreuses attestations - dont les dépêches précitées de Gaston Stiegler - , mais ne résiste pas aux reportages, et non des moindres, publiés après le retour de leurs auteurs ou témoignant simplement d'une décantation assez peu spontanée.

Sans prétendre trancher ici une question aussi incertaine, on peut émettre l'hypothèse qu'un repère essentiel sur l'essence $d u$ "grand» reportage se trouve dans le paradoxe fondateur qui le situe à la fois dans la continuité du reportage d'information journalistique et à l'exact opposé de celui-ci. On sait que la "révolution» déjà évoquée plus haut, la différenciation entre journalistes et écrivains ou essayistes au sein de la masse jusqu'alors indistincte des gens de lettres, est née de la prépondérance octroyée (ou plutôt rendue ${ }^{13}$ ) aux nouvelles, la recherche des faits l'emportant sur l'esprit et le style :

13 Le premier journal francophone, au XVIIe siècle, comme le premier quotidien au XVIIIe étaient tous deux consacrés aux nouvelles. 
Nous avons dit que les grands journaux donnaient peu de nouvelles, ajoutons qu'ils les donnent rarement fraîches et convenablement assaisonnées; des préoccupations d'un ordre plus élevé dont nous apprécions la portée et l'influence détournent leurs rédacteurs de ces soins assidus. (Le Petit journal, 1863, p. 1)

Ainsi les mérites des journalistes professionnels, les reporters, ne s'expriment-ils, au sein d'un processus ostensiblement industrialisé, qu'en termes de promptitude, d'abondance d'informations et, bien sûr, de véracité :

Le principal mérite du Figaro, il faut bien le dire, est d'être généralement mieux et plus rapidement informé que la plupart de ses confrères. [...] Le service de reportage spécial au Figaro, largement rétribué, commandé par un chef expérimenté, contrôlé par des sous-chefs intelligents, apporte chaque jour aux bureaux du journal un contingent de nouvelles de toutes sortes qui sont immédiatement examinées ; celles-là seulement, dont la véracité est bien prouvée, trouvent place dans les colonnes du journal. (Villemessant, 1873, p. 2)

La célèbre article dans lequel Hughes Le Roux oppose « le vieux journalisme et le nouveau » en 1887 (et non en 1888 comme l'indiquent notamment ${ }^{14}$ Boucharenc, 2004, p. 18, et Thérenty, 2007, p. 206) force assez le contraste entre «les vieux maréchaux de la chronique » et le reporter « à peine autorisé à signer d'une initiale ses articles sans prétention littéraire, uniquement préoccupé d'exactitude », pour qu'il soit nécessaire d'insister sur ces archétypes, largement attestés par ailleurs

\footnotetext{
14 Bien des auteurs (dont, confessons-le, celui de ces lignes) se sont à un moment ou à un autre appuyés comme elles sur Paul Ginisty, sorte de Violletle-Duc de l'histoire de la presse, dont l'Anthologie du journalisme a sauvé de l'oubli des textes importants au prix de restaurations audacieuses. Ainsi le titre "Le reporter », inventé par Ginisty, fait-il fi de la graphie "reporteur », ostensiblement employée par Le Roux dans l'article original mais « corrigée » par l'anthologiste.
} 
(voir, par exemple, Larousse, 1875, ou Filon, 1889). Indépendamment de leur réalité - les positions et pratiques effectives étant souvent moins tranchées -, ils soulignent combien le reportage, centré sur la nouvelle et le fait vérifié, symbolise le journalisme moderne, tant pour les professionnels que pour le public. Or, le développement en France de l'appellation de "grand reportage », dont les anglo-saxons n'ont jamais éprouvé le besoin, ne correspond pas à l'amplification quantitative (plus long, plus loin...) que l'adjectif aurait pu suggérer, ni simplement au besoin de se distinguer de la lie des « petits » reporters de faits divers. Tandis que le reportage avait consacré symboliquement le journalisme par l'exclusion du littéraire, le "grand reportage», en un retournement spectaculaire, célébrait au contraire les retrouvailles du journalistique et du littéraire. «Grand » vient donc rapprocher ce que "reportage» avait disjoint peu avant, mais cette réconciliation n'est possible qu'après que le journalisme s'est affirmé dans sa spécificité. Hughes Le Roux, dans son apologie du journalisme moderne, n'avait rien prophétisé d'autre, en annonçant qu'une fois disparues les «spirituelles élucubrations » des «hommes [...] du passé », le reportage

montera des bas-fonds du journal à la surface. Il tentera tous les gens de mérite, les artistes, les littérateurs, ceux qui savent voir, deviner, composer, écrire, ceux qui ont des impressions justes et profondes, ceux dont le regard perce les contours, va à l'âme des hommes et des choses. (loc. cit.)

Cette reconfiguration, cependant, n'a rien de simple, car elle ne dispense plus le talent de s'inscrire dans les normes symboliques qui caractérisent - en principe, sinon dans les faits - le journalisme nouveau. Ainsi peut-on bel et bien envisager le grand reportage en termes de distance, mais il 
s'agit de la distance que son auteur est habilité à prendre par rapport aux formes impersonnelles et objectivantes de l'écriture de presse "ordinaire » (embarrassés pour traduire l'expression française, les anglo-saxons recourent parfois à "reporter at large», qui en traduit bien l'idée). Cet écart dessine à sa façon le territoire du reportage : trop contraint, le récit n'est pas vraiment « grand »; trop désinvolte, il n'est plus du « reportage ».

\section{Du journalisme d'auteur?}

Percevoir le grand reportage comme doublement lié, par ses contraintes journalistiques d'un côté et ses ambitions auctoriales de l'autre, n'est pas radicalement nouveau en soi ${ }^{15}$ mais l'aborder comme une démarche fondamentalement antinomique conduit à s'interroger sur la nature de cette antinomie. Un grand reportage, après tout, ne pourrait-il être impeccablement journalistique et admirablement créatif et singulier?

Le canon même du "genre» alimente le doute. Peu de reporters, somme toute, ont assez marqué son histoire pour en être reconnus comme des maîtres (ou simplement pour être connus) en dehors du cercle des spécialistes. La même poignée de noms se dégage invariablement: Albert Londres et Joseph Kessel, bien sûr, et peut-être Lucien Bodard ou Henri Béraud. Or, ces parangons du grand reportage ont invariablement en commun, outre leur talent de plume, la «distance» qu'ils

\footnotetext{
15 Voir notamment l'opposition entre la «littérarité » et la «littéralité »du reportage, analysée par Boucharenc (2006), ou l'institutionnalisation du grand reportage dans les années 1930 étudiée par Aron (2012).
} 
pouvaient prendre avec la rigueur des faits. Au delà d'une subjectivité assumée (qui conduira Béraud à ses égarements collaborationnistes), c'est bien la véracité même - non pas de leurs récits mais de détails colorés de ceux-ci - qui a été mise en doute. Bodard, qui fascinait autant pour sa capacité à décrire une guerre sans presque quitter le bar de son hôtel que pour son style et ses intuitions, occupe sans difficulté un rôle à part, un peu canaille, dans l'imaginaire journalistique, mais le cas d'Albert Londres perturbe plus: ce que l'on accorde à Gargantua ne convient pas à Don Quichotte. D'où l'âpreté des échanges entre les gardiens de ce symbole et ceux qui, comme Jean-Pierre Tailleur ou Claude Moisy, lui opposent les principes journalistiques.

Exemplaire Albert Londres? Pas vraiment si l'on considère sa propension à confondre journalisme et littérature, information et fiction, militantisme et rigueur. [...] Ses emballements poétiques, sa relation fantasque avec les faits et ses dénonciations des injustices «à la Victor Hugo » peuvent avoir leurs vertus dans un autre ordre d'activité créatrice. Mais je les ai toujours considérés comme incompatibles avec le sain et rigoureux exercice de l'information. (Moisy, 2004, souligné par nous)

Force est de constater que, si rien n'interdit en théorie de marier dans le grand reportage le plus haut niveau des valeurs « auctoriales » et «journalistiques», une sorte de fatalité veut que les figures tutélaires du journalisme français ne paraissent s'être élevées dans les unes qu'au détriment, au moins partiel, des autres. Sans doute, leur notoriété même a pu conduire à examiner de façon plus tatillonne la fiabilité de leurs articles (à l'instar de ceux d'Hemingway ${ }^{16}$ ). Mais, en tout état de cause, ces

16 «excellent écrivain, Hemingway était déficient en tant que reporter» (Harrison, 1994, p. 11, notre traduction) 
écarts à la vérité pure ne remettent pas vraiment en cause leur place au panthéon du grand reportage. Car si l'on peut montrer que la promesse d'un certain type de vérité est le fondement même de l'épistémologie constitutive du journalisme (Labasse, à paraître) et, dans une perspective plus normative, qu'elle constitue «sa première obligation» (Kovach et Rosenstiel, 2001, p. 37), les accrocs à cet engagement complexe ne disqualifient pas nécessairement leurs auteurs. L'important est dans la revendication de ces principes, l'allégeance à l'ensemble diffus, modérément consensuel et parfois contradictoire de valeurs, de méthodes, d'attitudes et de traditions professionnelles qui forme la culture du journalisme, y compris lorsqu'il s'agit de contester celle-ci de l'intérieur.

Ici, Cocteau peut à nouveau fournir un précieux repère. On doit pour cela le rapprocher - au mépris des époques, des contextes et des continents - d'un autre jalon non moins déconcertant, Hunter $S$. Thompson, dont l'un des plus célèbres récits, «Las Vegas Parano », entre curieusement en résonance avec le sien (sous un prétexte quelconque, deux amis partent en voyage aux frais d'un journal. Assez peu soucieux de décrire ce qu'il était venu voir, le narrateur se préoccupe essentiellement de faire partager son ressenti de l'instant et son goût pour les errances nocturnes). L'un et l'autre marchent sur les traces d'un mythe (le «cœur du rêve américain » dans le second cas), l'un comme l'autre pourrait avoir écrit «L'admiration me laisse froid. Je veux des coups de foudre. La haine ou l'amour. ${ }^{17}$ mais, comparé aux extravagances d'un Thompson embrumé de LSD et de mescaline, le récit gentiment opiacé de Cocteau paraît infiniment plus proche d'un reportage, quel que soit le sens que

17 Ça ressemble à Thompson, mais c'est bien Cocteau (op. cit., p. 132). 
l'on veuille donner à ce terme. Pourtant, les divagations de l'Américain (violemment rejetées par le journal commanditaire mais accueillies par un autre) appartiennent sans nul doute à l'histoire mondiale du journalisme : dans leurs outrances, elles marquent ce point extrême que l'on a appelé avec fascination et effarement le "Gonzo journalism ». Le récit de Cocteau, lui, ne trouve pas même de place dans le volet français de cette histoire, comme s'en indigne Jean Touzot (1999, p. 9). C'est que, si Thompson a déploré jusqu'à la fin de n'être pas sorti du journalisme, Cocteau n'a jamais prétendu, imaginé ou souhaité en faire partie. Comme le soulignait à juste titre Thérenty, «plus que le contrat donné par le directeur du journal, c'est un ethos qui permet de différencier nettement voyageur et reporter » (2006, p. 102). Mais alors... « Cocteau journaliste»?

L'identité dessine la ligne mieux que les textes: si le Ragueneau ${ }^{18}$ de Cyrano de Bergerac n'est pas vraiment un poète, ce n'est pas parce que ses vers sont médiocres (on peut fort bien être un vraiment mauvais poète), c'est parce qu'il est essentiellement, foncièrement, un pâtissier. Les journaux, du reste, ne manquent généralement pas de caractériser leurs envoyés spéciaux allogènes. Ainsi L'Intransigeant accueille-t-il avec Marinetti «le premier récit d'une bataille moderne vécue et décrite par un poète» (1911, p.2), tandis que Malraux est dûment présenté comme le « Prix Goncourt 1933 » $(1934$, p. 1) et Saint-Exupéry comme «le célèbre pilote-écrivain » (1936, p. 1). Les bénéfices promotionnels de ces étiquettes sont évidents, mais celles-ci constituent aussi un avertissement clair : celui qui va écrire n'est pas tenu au respect des normes et usages professionnels (aussi élastiques que soient ceux-ci en France).

18 On parle ici du personnage, et non de son infortuné modèle. 


\section{Périls et vertus des distinctions}

Si floue que soit l'identité journalistique, les indices de sa revendication individuelle et les formes et degrés de sa reconnaissance constituent des repères importants pour lire n'importe quel écrit de presse, mais ces éléments deviennent essentiels lorsqu'il s'agit de "grand reportage», justement parce que celui-ci résulte de l'interaction, féconde mais contradictoire, entre deux ${ }^{19}$ ordres de valeur.

Méconnaître l'un d'eux au nom de l'autre peut conduire à la tentation (perceptible dans la note de Touzot supra) de corriger ex cathedra l'histoire du journalisme pour y inclure tel ou tel auteur révéré, ou même à des assertions renversantes, comme la croyance que nul ne se souviendrait de cette "Miss Bly» si elle n'avait eu "l'idée tout de même saugrenue de rivaliser avec un héros de roman ", affichée avec candeur par un spécialiste reconnu de Blaise Cendrars (Leroy, 2001, p. 145).

On voit ici que la délégitimation peut fonctionner dans les deux sens: si les écrivains sont peu mentionnés dans les monographies sur la presse (comme le remarque également Boucharenc, 2004, p. 12-13), les journalistes ne sont guère plus admis par le champ littéraire traditionnel qu'ils ne l'étaient au temps de la fameuse charge de Brunetière contre les livres de journalistes : «[...] voilà le reportage, et voilà sous quelle forme il est en passe, traîtreusement, de s'introduire, je ne dirai pas seulement dans le roman, je suis obligé de dire dans la littérature contemporaine. » (1883, p. 223)

Tandis que le passé journalistique d'un auteur ou ses

\footnotetext{
${ }^{19}$ Et parfois, si l'on y ajoute l'engagement politique, trois ordres de valeur.
} 
collaborations ultérieures ne sont en rien disqualifiants vis-àvis de la postérité - l'histoire littéraire s'en trouverait, sinon, singulièrement dépeuplée - c'est bien la posture identitaire qui dessine l'invisible frontière, comme si la consécration dans un ordre exigeait l'abjuration de l'autre ${ }^{20}$ ou s'il existait « une guerre non déclarée entre le journalisme et la fiction» (Underwood, 2013). De fait, si l'on excepte évidemment l'habile Kessel, le nombre des écrivains capables de susciter des études universitaires sans pour cela avoir renié le journaliste en eux (Roger Lemelin ? Sorj Chalandon ?) paraît étonnamment réduit face à tous ceux qui l'ont ostensiblement relégué au rang de marchepied ou d'apprentissage, souvent parce que c'était effectivement le cas mais souvent aussi pour prendre, bon gré mal gré, leurs distances avec une image professionnelle devenue un obstacle à leur reconnaissance littéraire :

Quand Ernest Hemingway travaillait comme reporter pour le Kansas City Star, le Toronto Star, et d'autres journaux pendant les années 20, ses expériences journalistiques ont été considérées comme un «apprentissage» pour son œuvre ultérieure, et son écriture a été rabaissée comme étant «juste du journalisme». Mais lorsqu'il réutilisa mot pour mot des portions du même matériel dans des romans, ce fut élevé à la dignité de littérature [...]. Pourquoi le journalisme est-il si difficilement apprécié au moment de sa création, avec tous ses problèmes, contradictions, limites et anomalies ? (Zelizer, 2004, p. 1 , notre traduction)

À l'évidence, la charge développée par Barbie Zelizer dans un ouvrage au titre éloquent (Prendre le journalisme au sérieux) est historiquement justifiée, mais elle n'en arrive pas moins une

\footnotetext{
20 Indépendamment, bien sûr, de leurs mérites propres. Le fait qu'une large part de l'imposante production livresque d'origine journalistique, hâtive ou triviale, paraisse mal armée pour résister à l'épreuve du temps ne suffit pas à expliquer le faible nombre des livres de journalistes accédant à la postérité.
} 
bonne décennie trop tard, en tout cas dans le champ des études littéraires francophones. Comme on le rappelait en introduction, la tour d'ivoire a ouvert ses portes - sans beaucoup de combats, si ce n'est d'arrière-garde - aux textes médiatiques. Est-ce à dire, à l'heure des premiers bilans, que toutes les distinctions se sont évanouies? Évidemment non, et c'est fort heureux, car on peut soutenir que la prise en compte de ces différences, plutôt que leur occultation, ouvre des perspectives encore plus vastes aux travaux sur la littérature médiatique.

Certes, les hiérarchies et les distinctions tendent à sauvegarder ce vieux tropisme lagarde-et-michardien de la dévotion aux Grands Auteurs, dont on évoquait plus haut quelques exemples, mais ce n'est pas un prix trop élevé à payer au regard de tous les éclairages que la question «En quoi ceci est-il différent de cela? » (par exemple le fait divers et l'art du roman, ou encore... Cocteau et le journalisme) peut encore apporter, tant à l'étude de la littérature qu'à celle des médias.

Ainsi en va-t-il de la question, familière et pourtant négligée, de la référentialité. Dès lors que, à quelques expériences littéraires près, tout texte vise d'une façon ou d'une autre à figurer un état possible du monde et de ses occupants (l'écrit se prêtant moins à l'abstraction que la peinture ou la musique), son degré de conformité avec ce qu'il entend représenter en constitue a priori une dimension fondamentale. Cependant, l'invention de la littérature au XIX siècle et la consécration de son triptyque «évident» (roman-poésiethéâtre) ont conduit à n'accorder que peu d'attention à cette dimension, comme si l'assimilation de la littérature à la fiction réduisait son rapport au réel à une considération anecdotique. 
Certes, on a assez moqué les mousquetaires de Dumas galopant au milieu des champs de pommes de terre, culture inconnue en Europe à leur époque, et Zola lui-même, tout arc-bouté qu'il fut contre l'idéologie romantique de l'imagination («Aujourd'hui, la qualité maîtresse du romancier est le sens du réel. »; 1880, p. 208), a dû se justifier :

Des critiques, éplucheurs de détails, après avoir gratté l'œuvre dans tous les sens, ont découvert que j'avais commis l'impardonnable anachronisme de mettre à l'horizon [...]. Je luttai d'abord pour l'amour des dates, mais ces masses étaient trop tentantes [...] j'ai succombé, et mon œuvre ne vaut certainement rien, si les lecteurs ne peuvent se résoudre à accepter cette erreur volontaire de quelques années dans les âges des deux monuments. (1884, p. 187)

Mais, au-delà de ces escarmouches récurrentes, le réel n'est le plus souvent considéré qu'en tant que source d'inspiration (untel et l'Afrique) ou en tant que thème (la représentation de la misère chez untel).

C'est de l'étude des mémoires et autobiographies qu'est venue la vive dénonciation, étonnamment récente, de ce "déficit théorique » face " aux res factae qui souffrent, pour la majorité, de se trouver dans l'angle mort de l'attention critique » (Jeannelle, 2004, p. 280). Les questions de distinction entre écriture fictionnelle et écriture du réel avaient certes soulevé occasionnellement un intérêt théorique, notamment chez Genette (1979/2004), qui soulignait que la narratologie avait « consacré une attention presque exclusive aux allures et aux objets du seul récit de fiction [...] comme en vertu d'un privilège implicite qui hypostasie le récit fictionnel en récit par excellence, ou en modèle de tout récit » (p. 141), mais sans que cette attention ne connaisse de postérité significative. 
Toutefois, le cas des textes médiatiques incite à aller plus loin que celui des mémoires en avançant que la référentialité, plutôt que de tracer une discontinuité, constitue bel et bien une "dimension» globale de l'écrit (au sens ordinal du terme). Échappant aux cadrages a priori, cet angle permet en effet de considérer la totalité des textes comme un continuum étagé par leur rapport au réel. Dans un premier temps, les catégories usuelles paraissent s'accorder sans trop de difficulté avec cette perspective, mais seulement sous leurs manifestations les plus typiques (soit, du plus au moins référentiel, les travaux scientifiques, les écrits journalistiques, les textes d'idées, la fiction réaliste, la fiction fantastique, etc.). Bien des textes brouillent ces frontières et certains inversent même les positions. Ainsi la Tentative d'épuisement d'un lieu parisien par Perec, dans sa sèche platitude, dépasse-t-elle en précision référentielle n'importe quel récit journalistique. De surcroît, certaines des classifications les mieux établies peuvent couvrir la totalité du spectre : c'est évidemment le cas des mémoires et autobiographies, mais ce peut être aussi celui du théâtre ${ }^{21}$ et, plus encore, de la poésie. Si la poésie scientifique et l'actualité en vers, florissantes à l'époque classique, ne subsistent guère qu'à l'état de traces, les textes (articles? poèmes ? éditoriaux ?) consacrés à la guerre par Coteau dans la revue Le Mot ou les figurations de Marinetti sur le même thème témoignent de leur persistance contemporaine. Mais au bout du compte, n'est-ce pas la majeure partie de la poésie qui relève, comme le journalisme, de la «non-fiction », par exemple lorsqu'elle vise à traduire les sentiments éprouvés à l'égard d'une personne, d'un paysage ou de n'importe quelle autre chose «existant»?

21 On peut songer par exemple aux « reconstitutions ». 
Décidément, ce n'est pas dans la fiction que la littérature pourra trouver le secret de sa littérarité, la fiction n'en étant, tout au plus, qu'un cas particulier.

Plongeons résolument dans l'abîme qui s'entrouvre : cette fiction ne serait-elle, au bout du compte, qu'une facilité ? Comme le remarque Pierre Bayard (2011) - lequel n'hésite pas à rapprocher les meilleurs auteurs et Jayson Blair, le journaliste-faussaire du New York Times -, imaginer un lieu est moins fatigant que s'y rendre pour de bon. De même, inventer des personnages et des situations réclame sensiblement moins de temps et d'énergie qu'il n'en faut pour enquêter, inventorier scrupuleusement la réalité et se soumettre à son joug, souvent si terne. Évidemment, le réel, bien souvent, n'est pas à la hauteur de la fiction : si triste qu'il soit, le suicide de Delphine Delamare est moins intéressant que celui d'Emma Bovary. De plus, la référentialité ne se limite pas à la factualité : la "vérité » des personnages et des scènes, y compris imaginaires, dépasse évidemment la question de leur existence effective (sans pour autant échapper en rien aux contraintes référentielles ${ }^{22}$ ). C'est à ce titre que toute fiction peut se dire un discours sur le monde réel, porteur de vérités qui, pour n'être pas factuelles, n'en sont pas moins profondes. Mais c'est à ce même titre que Bodard, comme bien d'autres, justifiait ses bidonnages journalistiques :

La réalité, qu'est-ce que c'est ? Une chose très subjective. Tout ce qu'on sent dans un pays, ce sont des potentialités, il s'agit de

\footnotetext{
22 Les jugements critiques sur le caractère plus ou moins « invraisemblable», « artificiel », « stéréotypé », " simpliste », etc., de personnages ou de situations romanesques témoignent de la persistance de ces contraintes jusque dans les univers les plus fictionnels.
} 
les rendre et c'est une question de talent. Où est la frontière entre le subjectif et l'objectif, quel est le dosage exact? c'est assez difficile à dire. (1970, p. 38)

Or, ce n'est pas seulement une «question de talent»: toutes choses égales par ailleurs (circonstances, intrigue, écriture, etc.), un récit présenté comme véridique aura toujours plus de poids qu'un récit inventé. Pour poignant que soit le jeune rescapé des massacres de Chio imaginé par Hugo, il le serait évidemment plus s'il avait été réel. À l'inverse, le journal d'Anne Frank resterait bouleversant sous la plume d'un écrivain contemporain, mais beaucoup moins. La réalité n'est pas un détail. Elle possède une puissance qui lui est propre et, comme le coût de cette puissance est élevé en termes de contraintes narratives, la tentation de la revendiquer sans en acquitter le prix est constante, ainsi qu'en attestent, par exemple, les dénonciations récurrentes qui visent les mémoires embellis ou fabriqués (Henri Charrière, Misha Defonseca, James Frey, etc.)

En somme, l'œuvre idéale, à la fois impeccablement factuelle et admirablement créative, reste un Everest encore inviolé pour la littérature comme pour le journalisme.

\section{Variabilité du sens des réalités}

Une prise en compte plus attentive de la factualité comme l'une des dimensions fondamentales de l'univers de l'écrit contribue à rejeter la dichotomie implicite mais prévalente qui oppose des journalistes maîtres des faits et des écrivains régnant sans partage sur l'imagination et le style. Contrairement à l'identité littéraire ou journalistique, et bien qu'il soit par ailleurs un élément essentiel de cette dernière, le rapport au réel constitue 
moins une démarcation corporative entre journalistes et écrivains qu'une frontière épistémologique entre les écriveurs ${ }^{23}$ en général et la réalité du monde qu'ils traduisent en mots. C'est pour n'avoir pas réellement perçu son importance que la sphère francophone, voire latine, traditionnellement désinvolte en la matière (se non è vero è ben trovato) ${ }^{24}$, demeure si en deçà des dynamiques qu'elle a suscitées sous d'autres cieux. À l'inverse, en effet, le monde anglo-saxon - justement parce qu'il est le plus attentif à cette frontière ( « facts are sacred») est celui qui a le plus vigoureusement et systématiquement entrepris l'exploration des richesses, y compris littéraires, qui s'étendaient au-delà. Ainsi, le berceau du journalisme positiviste et du fact-checking était aussi, dans les années 1960, celui du New Journalism, un réinvestissement de l'écriture du réel où se mêlaient journalistes et écrivains, et dont Tom Wolfe n'hésitera pas à affirmer qu'il a «causé la panique, détrônant le roman comme le premier des genres littéraires, ouvrant la première direction nouvelle dans la littérature américaine en un demi-siècle » (1972, p. 1, notre traduction).

Sans débattre ici de la rigoureuse factualité d'une telle

\footnotetext{
23 L'emploi antérieur d' "écrivants», que Barthes (1964) oppose à «écrivains", contraint à se rabattre sur cet hyperonyme malsonnant pour désigner à la fois les uns et les autres sans recourir à l'appellation de «gens de lettres », décidément désuète.

24 L'article d'un critique littéraire du nouvel Observateur sur les affabulations reprochées à un auteur américain en est révélateur jusqu'à la caricature (volontaire ?) : "en réalité, Mortenson ne serait allé à Korphe qu'un an après sa tentative de gravir le K2. Contrairement à ce qu'il prétend, il n'aurait pas été kidnappé par des talibans en 1996. Le lecteur indulgent pour les errements de la vanité humaine dira: et alors? Étonnamment, ce sont ces petits arrangements avec la vérité qui semblent, à la lecture de la presse américaine, avoir le plus choqué l'opinion. Ce n'est pas une surprise : on sait depuis longtemps maintenant que les Américains n'aiment pas les mensonges, même petits, même pieux. » (Caviglioli, 2011)
} 
affirmation, ni même de la réalité de la bannière commune sous laquelle ce manifeste enrôlait aussi bien Norman Mailer que Truman Capote et bien d'autres, force est de constater que le nouveau monde a autrement mieux réalisé que l'ancien la prophétie séculaire d'Hughes Le Roux (supra) pour qui le reportage ne manquerait pas de conquérir «les littérateurs, ceux qui savent voir, deviner, composer, écrire [...] ceux dont le regard perce les contours, va à l'âme des hommes et des choses ». Affaibli par les libertés prises par certains auteurs avec les faits, le New Journalism a en effet été relayé par la mouvance plus vaste de la Creative Nonfiction, dont l'expansion ne se dément pas dans le champ éditorial comme dans l'enseignement universitaire et même scolaire (au point de soulever des protestations ${ }^{25}$ ). Mais en réalité, les racines de ces approches du réel sont beaucoup plus profondes qu'on ne l'imagine traditionnellement. L'idée, bien ancrée depuis la fin $\mathrm{du} \mathrm{XIX}^{\mathrm{e}}$ siècle, selon laquelle le journalisme américain ne connaissait que la plate sécheresse des reportages factuels, laissant aux Français le raffinement de la plume et des idées, pouvait certes rassurer les professionnels qui la soutenaient alors (Fernand Xau, Pierre Giffard, etc.), mais la persistance de cette croyance française est curieuse si l'on considère que le Literary Journalism a commencé à devenir une forme foisonnante et critiquement reconnue à partir de la fin de la

25 Par exemple celles de Douglas Hesse qui, il y a plus de vingt ans déjà, s'interrogeait sur la valeur pédagogique de ces formes non canoniques, mais aussi sur les motivations de leurs promoteurs : "Pour le dire crûment, dans un paysage surchargé d'universitaires, un moyen de gagner de l'espace d'expression est de déclarer de nouveaux territoires ouverts à la colonisation. Tout à coup, il n'y a plus seulement des textes vierges à explorer, mais un genre vierge, pas seulement des pays mais des continents. [...] Déclarer ce territoire en affirmant la littérarité de l'essai est un geste qui comporte des profits collectifs autant qu'institutionnels. » (1991, p. 324, notre traduction) 
guerre de Sécession (voir Hartsock, 2001), qu'il a ensuite régné dans des magazines aussi lus qu'Atlantic Monthly, le New Yorker ou Esquire bien avant que l'on n'évoque le New Journalism ou la Creative Nonfiction, et qu'il n'a nullement disparu depuis, d'où le chaos actuel des désignations alternatives et des définitions entrecroisées.

Ce chaos définitionnel, qui continue à susciter aux ÉtatsUnis nombre de débats critiques et universitaires, est sans doute déconcertant, mais moins déconcertant que l'indifférence réservée par la plupart des chroniqueurs parisiens et des départements de littérature française au vague fourre-tout conceptuel que les libraires (qui, eux, ont besoin d'une étiquette) ont nommé «Essais et documents». Par une suprême ironie, le colloque annuel de l'International Association for Literary Journalism Studies s'est tenu plusieurs fois en France, mais à l'initiative de départements d'études anglophones, sans qu'un auteur français n'y soit abordé.

En somme, l'antique mission de gardiens de l'ineffable, assignée par Sainte-Beuve, paraît toujours dominer les études de lettres françaises :

deux littératures coexistent dans une proportion bien inégale et coexisteront de plus en plus, mêlées entre elles comme le bien et le mal en ce monde, confondues jusqu'au jour du jugement : tâchons d'avancer et de mûrir ce jugement en dégageant la bonne et en limitant l'autre avec fermeté (1839, p. 691).

On pourrait évidemment multiplier les exemples de transgression par rapport à cette feuille de route, dont le plus évident est la prise en compte, de moins en moins timide, de la « paralittérature » (sic) fictionnelle dans les études littéraires. Il n'en resterait pas moins que l'écriture du réel demeure, pour 
l'essentiel, hors de la zone d'attention francophone - et surtout française - si ce n'est, à la rigueur, sous des formes égocentrées, légitimées par la notoriété culturelle de certains de leurs auteurs (telles que les écrits de voyage, mémoires, journaux intimes et correspondances, compléments usuels d'une œuvre romanesque reconnue). Il suffit, en revanche, d'essayer quelques mots clefs comme «creative nonfiction» dans un catalogue de librairie en ligne pour se faire, par comparaison, une idée assez juste de l'insouciance francophone qui frappe toujours le champ de l'écriture d'enquête ou d'observation. Un domaine qui, dans une langue, déchaîne une offre éditoriale de plusieurs centaines d'ouvrages (manuels, anthologies, traités, etc.) continue à ne rencontrer, dans une autre, qu'un quasi-silence. Si «littérature» est synonyme de «fiction», cette lacune éditoriale n'est qu'anecdotique. Si ce n'est pas le cas, elle est le signe, ou plutôt l'un des signes ${ }^{26}$, d'un dénuement assez embarrassant. D'où l'importance du renouveau qui s'est presque simultanément amorcé en Belgique (à partir des études sur le journalisme ${ }^{27}$ ) et en France (à partir des études littéraires). Mais d'où l'importance, aussi, des difficultés qu'il reste à surmonter.

\section{Littérature, où es-tu?}

Le problème essentiel réside dans la construction des objets, à la croisée de perspectives disciplinaires différentes, mais aussi à celle de cultures professionnelles hétérogènes. Les défis de désignation (nonfiction créative, journalisme littéraire, etc.) qui,

\footnotetext{
${ }^{26}$ Le nombre de cours d'analyse ou de création littéraire consacré à ce « genre » (?) en est un autre.

27 Voir Lits (2008), Lallemand (2011), Meuret (2012), Vanoost (2013), etc.
} 
en français comme en anglais, guettent tout travail en la matière en sont le témoin, voire le centre. Or, ils sont exactement de même nature que la perplexité que requiert un titre comme Cocteau journaliste. L'exemple presque idéal que fournit le poète incite ainsi à généraliser les réflexions qu'il a alimentées plus haut et à différencier formellement les objets, concentriques, de la nonfiction et du journalisme. Dans cette perspective, le journalisme, quelque épithète qu'on lui accole, décrit une pratique définie par son projet (il n'y a pas de journaliste involontaire) et situé par rapport à une culture professionnelle (y compris en se confrontant à celle-ci). La nonfiction, pour sa part, renvoie à un domaine beaucoup plus large et plus graduel, défini seulement par son rapport à la factualité. La première catégorie est culturelle; la seconde est épistémologique. La première tend à séparer reporters et littérateurs; la seconde les réunit au sein d'un continuum textuel : aborder de façon distincte la « journalistitude ${ }^{28}$ d'un auteur (disons... Cocteau) et la «factualité » de ses récits ne conduit donc pas aux mêmes observations.

Reste, évidemment, le troisième terme de l'équation décrivant les écritures du réel, la décourageante énigme de la « littérarité ». Si celle-ci ne faisait guère de doute dans le cas du poète, elle paraît beaucoup plus discutable dans le cas de la plupart des textes journalistiques ordinaires, c'est à dire éphémères ("La littérature est le contraire du journalisme", assénait ainsi Gide, 1993, p. 18). Toutefois, comme le souligne Alain Vaillant, « il faut inverser la perspective et se demander, de manière bien plus radicale, comment un texte médiatique -

28 Une fois encore, le lexique disponible montre ses limites, contraignant à ce désastreux barbarisme. 
donc voué par nature à la plus large diffusion publique pourrait ne pas être littéraire» (p. 22-23). La question est d'autant plus intéressante que ses enjeux dépassent largement son objet originel. En se confrontant aux textes médiatiques, le champ littéraire peut éclairer non seulement ceux-ci mais aussi sa propre essence. Mais le risque demeure, suivant en cela un tropisme familier, de ne prélever dans le journalisme que les morceaux nobles - les textes de «vrais» auteurs (Colette, Cendrars, etc.) - et, plus généralement, les «beaux textes» (chroniques, grands reportages, tribunes, etc.). Alain Vaillant lui-même, après avoir retranché les plus bas morceaux - les textes purement pratiques tels que la météo - ne concède au reste qu'une « littérarité précaire et conditionnelle » (p. 26) :

La plupart de ces nouvelles, prises dans le flux continuel de l'actualité où les événements du jour recouvrent et effacent ceux de la veille, n'ont de validité que pour le jour même de leur publication, ne concernent que les lecteurs de ce jour précis. [...] l'article d'information n'est destiné qu'à une lecture immédiate et ponctuelle, alors que le texte littéraire, par sa nature communicationnelle, est censé avoir la capacité de durer. (ibid.)

Pour autant, ces textes posent aux belles-lettres la question même (symétriquement inversée) que le tour du monde de Cocteau posait au reportage: en quoi suis-je différent? D'où l'on inférera, en première analyse, que la « journalistitude » (resic) et la "littérarité » sont le même genre de chose: une considération sociale, fondée sur les valeurs propres à un champ. Ce n'est pas bien nouveau, mais il n'est pas inutile de poursuivre le parallèle. La question du journalisme avait amené à distinguer cette catégorisation sociale d'une caractérisation plus englobante fondée sur la factualité des textes eux-mêmes. Or, le champ littéraire connaît lui aussi un mode de 
caractérisation élargi, graduel et fondé sur les textes: leur poétique, évidemment (au sens moderne du terme: l'agencement spécifique du langage et non l'élévation du style). De même que le centrage sur la fiction avait maintenu dans l'ombre la dimension essentielle que constitue la référentialité de toute œuvre, de même peut-on noter que l'invocation de l'actualité - avec tout ce qu'elle implique de périssable - a généralement $^{29}$ dispensé de se pencher sur la poétique des textes journalistiques ordinaires.

$\mathrm{Si}$, comme on l'a suggéré plus haut, l'apport le plus fécond de l'approche littéraire aux abondants travaux préexistants sur l'histoire et la sociologie du journalisme est bien d'amener à lire les articles de presse en tant que textes - c'est-à-dire "de prendre la presse comme un objet littéraire à part entière, relevant des méthodes de poétique, de rhétorique, voire de linguistique traditionnellement mises en œuvre pour l'étude des autres formes génériques» (Thérenty, 2003, p. 630) encore faut-il les lire tous. Un examen attentif permettrait par exemple de réaliser que ces écrits cachent, derrière le dénuement stylistique revendiqué par la plupart des professionnels $^{30}$, des performances narratives proprement extraordinaires. On sait que le procédé type de construction du récit journalistique repose sur la structuration dite «en pyramide inversée» (voir Labasse, 2012). Développée au XIXe siècle aux États-Unis et toujours largement employée, cette forme suppose que les premières lignes condensent l'essence de l'information (qui a fait quoi, quand, où, pourquoi, comment) puis que les éléments se succèdent du plus important au moins

${ }^{29}$ Les faits divers criminels, par la fascination qu'ils exercent, ont cependant suscité l'intérêt de divers auteurs (R. Barthes, D. Kalifa, etc.)

30 « Le journalisme n'est pas de la littérature » (Voirol, 1992 p. 15). 
important. Pour les étudiants en journalisme et les plus traditionnels de leurs professeurs, il s'agit de la façon «normale » de décrire et raconter :

L'écriture journalistique ne peut connaître qu'un seul plan [...]. Le premier paragraphe de tout article doit dire le cœur de l'affaire. La suite les développe par ordre d'importance décroissante. Sans doute, les modalités de cette écriture-là sont très variées [...]. Mais la construction est invariable. (Mouriquand, 1997, p. 48)

Il suffit pourtant de soumettre des étudiants en lettres au même exercice pour vérifier, à l'aune de leur désarroi, combien cette construction "normale » est inouïe : ni l'Oulipo ni le Nouveau Roman n'ont imaginé un tel dispositif. Et il ne s'agit pas là d'une exploration expérimentale ou d'un cauchemar d'écrivain, mais de la forme ordinaire des nouvelles, telles qu'elles sont chaque jour publiées dans les journaux et sur Internet. Ainsi peut-on envisager le récit journalistique comme une littérature à contraintes multiples, non seulement formelles (comme la structuration ou encore l'astreinte aux mots courts et aux phrases brèves) mais aussi et surtout référentielles (factualité, impartialité, recoupement, etc.). Pour autant, comme l'admet Mouriquand, ses modalités sont «très variées »: même pour ceux qui s'en tiennent au moule de la pyramide inversée, le choix premier de l'agent (qui ?) de l'action (quoi ?) et des autres éléments circonstanciels permet, pour une même information, de multiples combinaisons et le classement de la suite par ordre d'importance confronte à une variété de possibilités plus vertigineuse encore. Il paraît difficile, dès lors, de leur dénier un intérêt narratif réel sans le dénier du même coup à une bonne partie de la littérature du XXe siècle. Mais la mosaïque des articles effectivement issus de ces normes n'a guère été étudiée, 
pas plus que ne l'ont été ceux qui s'en écartent et bien d'autres aspects et formes par lesquels le journalisme inscrit dans le texte la « réalité » du monde.

Les articles de presse attendent toujours qu'on les lise vraiment, tant pour eux-mêmes que pour les éclairages qu'ils peuvent apporter par contraste aux récits littéraires: se concentrer sur les «grands auteurs » et les «textes nobles » du journalisme - se contenter, en un mot, d'élargir un peu les frontières de l'histoire littéraire - serait renoncer aux profits épistémologiques majeurs que recèlent encore les questions symétriques du journalisme comme objet littéraire et de la littérature comme approche du journalisme.

\section{Conclusion}

Cocteau n'est pas journaliste. C'est la remarque que suscite, avant même de l'ouvrir, le recueil (par ailleurs remarquable) publié récemment sur ce thème. Et pourtant, le rapprochement inattendu qu'opère son titre est particulièrement stimulant, le réfuter ou le soutenir étant également délicat.

À l'instar de la fiole de colorant versée par des hydrographes dans un réseau fluvial, la dilution des textes de presse du poète dans la vaste mouvance du récit journalistique peut permettre d'observer des sinuosités, des perméabilités et des diffluences plus ou moins visibles en temps ordinaire. C'était, en tout cas, l'approche adoptée par cet article.

Suivre ce repère dans quelques-uns de ces méandres a ainsi conduit à avancer plusieurs remarques et propositions sur les rapports entre le journalisme et la littérature en tant que 
constructions sociales, que pratiques textuelles et qu'objets de recherche. Son propos, on l'a dit, n'était pas de démontrer formellement une thèse principale, mais plutôt de contribuer à illustrer les riches perspectives qu'ouvre la rencontre des travaux sur ces deux champs. En s'interrogeant mutuellement, ils tendent en effet à exposer non seulement leurs propriétés formelles mais, plus généralement, leur façon d'être et même leur raison d'être.

\section{Bibliographie}

AlBerT, P. (1972), «La presse française de 1871 à 1940 », dans C. Bellanger et al. (dir.), Histoire générale de la presse française, t. III, Paris, Presses universitaires de France, p. 447-622.

ARON, P. (2012), «Entre journalisme et littérature, l'institution du reportage », Contextes, no $11, s . p$.

BARTHES, R. (1964), Essais critiques, Paris, Seuil.

BAYARD, P. (2011), Comment parler des lieux où l'on n'a pas été ?, Paris, Éditions de Minuit.

Bly, N. (2009), Around the World in Seventy-Two Days, Rockville, Wildside Press.

BODARD, L. et J.-C. ZYLBERSTEIN. (1970), « Bodard à la question », Le nouvel Observateur, no 274, 9 février, p. 38.

Boucharenc, M. (2006), "Choses vues, choses lues: le reportage à l'épreuve de l'intertexte», Cahiers de Narratologie, no 13 , s. p. 
-. (2004), L'Écrivain-reporter au cour des années trente, Villeneuve d'Ascq, Presses universitaires du Septentrion.

BRUnetiere, F. (1883), Le Roman naturaliste, Paris, C. Lévy.

Camus, A. (1962), Carnets 1 ; mai 1935 - février 1942, Paris, Gallimard.

CAViglioli, D. (2011), «Qui veut mentir dans un best-seller et gagner des millions ?», Le nouvel observateur, 21 avril.

ChAPLIN, Charlie. (2012), My autobiography, New York, Melville House.

Cocteau, J. (1960), Poésie critique II, Paris, Gallimard.

-. (2009), Tour du monde en 80 jours, Paris, Gallimard.

DE BRoucKer, J. (1995), Pratique de l'information et écritures journalistiques, Paris, Éditions du CFPJ.

DE Chateaubriand, F.-R. (1812), Itinéraire de Paris à Jérusalem et de Jérusalem à Paris, en allant par la Grèce, et revenant par l'Égypte, la Barbarie, et l'Espagne, [seconde édition], Paris, Le Normant.

DE VILLEMESSANT, H. (1873), "Les informations du Figaro », Le Figaro, n- 81, 22 mars, p. 2.

FILON, A. (1889), "Courrier littéraire », Revue politique et littéraire (Revue bleue), no 12, p. 378-381.

GenetTe, G. (2004), Fiction et diction. Paris, Seuil.

GIDE, A. (1993), Conseils au jeune écrivain ; De l'influence en littérature, Paris, Proverbe.

GINISTY, Paul. (1920 et 1922), Anthologie du journalisme. Du XVIIe siècle à nos jours, 2 vol., Paris, Delagrave. 
HARRISON, S. L. (1994), « Hemingway as negligent reporter: New Masses and the 1935 Florida hurricane », American Journalism, vol. 11, no 1, p. 11-19.

HARTSOCK, J. C. (2001), A History of American Literary Journalism. The Emergence of a Modern Narrative Form, Amherst, University of Massachusetts Press.

HESSE, D. (1991), «The Recent Rise of Literary Nonfiction: A Cautionary Assay », Journal of Advanced Composition, vol. 11, no 2, p. 323-333.

Heron, P.-M. et M.-É. Therenty (dir.). (2014), Cocteau journaliste, Rennes, Presses universitaires de Rennes.

JEANNELlE, J.-L. (2004). «L'acheminement vers le réel. Pour une études des genres factuels : le cas des Mémoires », Poétique, no 139 , p. 279-280.

Kalifa, D. et A. VAillant. (2004), Pour une histoire culturelle et littéraire de la presse française au XIX ${ }^{\mathrm{e}}$ siècle, Le Temps des médias, no 2, p. 197-214.

- et al. (dir). (2012), La Civilisation du journal: Histoire culturelle et littéraire de la presse française au XIXe siècle (1800-1914), Paris, Nouveau Monde.

Kovach, B. et T. RosenstiEL. (2001), The Elements of Journalism, New York, Three Rivers Press.

L'INTRANSIGEANT (1911), "La guerre. Nouveaux combats en Tripolitaine ", L'Intransigeant, nำ 11485, 25 décembre, p. 2.

-. (1934), «Demain: À la découverte de la capitale mystérieuse de la reine de Saba », L'Intransigeant, no 19906, 2 mai, p. 1. 
—. (1936), « Espagne ensanglantée », L'Intransigeant, no 20734, 11 août, p. 1.

LABASSE, B. (à paraître), «Du journalisme comme une mésoépistémologie », Communication, vol. 33, nํㅜ 1 .

- . (2012), «Structures narratives et congruence cognitive : cas du summary lead et de la pyramide inversée ", Rédactologie / Canadian Journal for Studies in Discourse and Writing, vol. 24, no 1, p. 65-83.

LALLEMAND, A. (2011), Journalisme narratif en pratique, Bruxelles, De Boeck.

LaRousSe, P. (1875), "Reporters », dans Grand Dictionnaire universel du XIXe siècle, t. 13, p. 993.

Le Matin (1884), «Au lecteur », Le Matin, no 1, 26 février, p. 1.

Le Petit Journal. (1863), "Paris», Le Petit Journal, numéro spécimen, p. 1.

Le Roux, H. (1887), « La vie à Paris », Le Temps, no 9540, 18 juin, p. 2.

LerouX, G. (1901a), «Gaston Stiegler», Le Matin, no 6303, 25 mai, p. 1.

—. (1901b), «L'arrivée à Paris », Le Matin, no 6368, 2 août, p. 12.

LEROY, C. (2001), «Emmène-moi autour du monde!... ou Comment Phileas Fogg est devenu reporter», dans M. Boucharenc et J. Deluche (dir.), Littérature et reportage, Limoges, PULIM, p. 139-149.

LITS, M. (2008), Du récit au récit médiatique, Bruxelles, De Boeck. 
MARTIN, M. (2007), « Le voyage du grand reporter, de la fin du XIXe siècle aux années 1930 », Le temps des médias, $\mathrm{n}^{\circ}$ 7, p. 118-129.

MEURET, I. (2012), " Le journalisme littéraire à l'aube du XXIe siècle: regards croisés entre mondes anglophone et francophone », Contextes, no 11, s. p.

MoISY, C. (2004), "Albert Londres, le mauvais maître », Médias, no 2 , septembre.

MoURIQUAND, J. (1997), L'Écriture journalistique, Paris, Presses universitaires de France.

SAinte-Beuve, C.-A. (1839), « De la littérature industrielle», Revue des Deux Mondes, nº 19, p. 675-691.

STIEgLER, G. (1901a)., "Le tour du monde... en combien de temps ?», Le Matin, no 6286, 12 mai, p. 1.

—. (1901b), «Le tour du monde en X... jours ; Les premières étapes », Le Matin, no 6307, 2 juin, p. 1.

-. (1901c), "Le tour du monde en 63 jours», Paris, Société française d'imprimerie et de librairie.

Therenty, M.-È. (2003), "Pour une histoire littéraire de la presse au XIX ${ }^{\text {e }}$ siècle», Revue d'Histoire Littéraire de la France, no 103, p. 625-635

—. (2006), «Les "vagabonds du télégraphe" : représentations et poétiques du grand reportage avant $1914 »$, Sociétés \& Représentations, no 21, p. 101-115.

—. (2007), La Littérature au quotidien. Poétiques journalistiques au XIX' siècle, Paris, Seuil.

Thompson, H. S. (1977), Las Vegas parano : une équipée sauvage 
au cour du rêve américain, trad. Philippe Mikriammos, Paris, H. Veyrier.

TouzoT, J. (1999). « Portrait d'un "animal très bizarre"» : l'écrivainjournaliste », Littératures contemporaines, no 6, p. 9-14.

UndERWOOD, D. (2013), The Undeclared War between Journalism and Fiction. Journalists as Genre Benders in Literary History, New York, Palgrave Macmillan.

VAILlant, A. (2001), «Préface », dans M.-È. Thérenty et A. Vaillant (dir.), 1836, l'an I de l'ère médiatique. Analyse littéraire et historique de La Presse de Girardin, Paris, Nouveau Monde éditions, p. 6-14.

-. (2011), « De la littérature médiatique», Interférences littéraires, $\mathrm{n}^{\circ}$ 6, p. 21-33.

VANoosT, M. (2013), "Journalisme narratif: proposition de définition, entre narratologie et éthique », Les Cahiers du journalisme, nํㅡㄹ 25, p. 140-161.

VENAYRE, S. (2007), « Le voyage, le journal et les journalistes au XIX" siècle », Le Temps des médias, no 8, p. 46-56.

VoIroL, M. (1992), Guide de la rédaction, Paris, Éditions du CFPJ. Wolfe, T. (1972), «The birth of 'the new journalism'; eyewitness report by Tom Wolfe », New York Magazine, vol. 4, n- 7, 14 février, p. 1 et 30-45.

ZELIZER, B. (2004), Taking journalism seriously: News and the academy, Thousand Oaks, Sage Publications.

ZoLA, É. (1880). Le Roman expérimental, Paris, G. Charpentier.

-. (1884). "Une page d'amour», Le Figaro, Supplément littéraire, no 47, 22 novembre, p. 187. 


\title{
Résumé
}

Un nombre croissant de travaux franchissent le fossé qui s'est creusé au XIXe siècle entre le journalisme et la littérature. Mais s'ils offrent de nouvelles visions des champs qui s'étendent de l'un et l'autre côté de cette délimitation passablement floue, ils soulèvent aussi des difficultés, qu'un ouvrage récent consacré à «Cocteau journaliste» rappelle opportunément. Saisissant cette occasion, le présent article se propose, à partir du cas d'école que constitue le " journalisme » de Cocteau, d'examiner plus généralement certaines des questions définitionnelles et des perspectives stimulantes que recèle encore l'approche littéraire des textes journalistiques.

\begin{abstract}
A growing number of studies cross the nineteenth century ditch between journalism and literature. But, while they offer new views of the fields that extend from either side of that blurred boundary, they also raise difficulties, as a recent book on "Cocteau journalist" calls to mind. Taking this opportunity, this article intends, from the perfectly ambiguous case of the "journalism" of Cocteau, to examine more generally some of the definitional issues and challenging perspectives involved in the literary approach of journalistic texts.
\end{abstract}

